\title{
Human Plasmodium vivax mosquito experimental transmission
}

\author{
David J. Sullivan and Peter Agre
}

Johns Hopkins Malaria Research Institute, W. Harry Feinstone Department of Molecular Microbiology and Immunology, Johns Hopkins Bloomberg School of Public Health, Baltimore, Maryland, USA.

\begin{abstract}
Plasmodium vivax bench research greatly lags behind Plasmodium falciparum because of an inability to culture in vitro. A century ago, intentionally inducing a malaria infection was a strategy commonly used to cure late-stage syphilis. These controlled human malaria infections were used with expertise and persisted to the end of World War II. While controlled malaria liver-stage infection has been achieved for both $P$. vivax and $P$. falciparum, controlled human transmission to mosquitoes falls short for both species. In this issue of the $J \mathrm{Cl}$, Collins et al. present groundbreaking work that establishes a system to transmit $P$. vivax gametocytes from humans to mosquitoes. The authors injected a unique human isolate of $P$. vivax that reached high gametocyte density within weeks. This study provides a technical advance that will facilitate the study and eradication of the human parasite $P$. vivax.
\end{abstract}

The problem of $P$. vivax malaria Widespread attention to malaria in sub-Saharan Africa caused by Plasmodium falciparum obscures the importance of malaria in India, southeast Asia, and in the Americas caused by Plasmodium vivax. In the Americas, $75 \%$ of the malaria cases result from $P$. vivax, whereas $53 \%$ of the global burden of $P$. vivax is in Southeast Asia, with India alone representing $47 \%$ of the global $P$. vivax cases. In 2018, WHO reported 7.5 million $P$. vivax clinical cases (95\% CI: 5.9 to 9.3 million), in contrast to 228 million $P$. falciparum cases (1). However, other reports estimate that the $P$. vivax burden is closer to 15 to 20 million, with subclinical cases pushing those numbers even higher (2-4). Globally, $P$. vivax cases are increasing in countries where $P$. falciparum cases are decreasing, and some countries have only $P$. vivax (1). In Africa, a substantial number of $P$. vivax cases occur in Eastern countries bordering the Indian Ocean, whereas $P$. falciparum is reported to make up $100 \%$ of reported cases, and $P$. vivax is present at only very low levels, in largely Duffy-negative parts of Africa (3).

$P$. vivax infections are also serious and sometimes lethal $(5,6)$, and are more genetically (7) and geographically diverse $(8,9)$ than those of $P$. falciparum. Since the 1970s, research and knowledge about $P$. vivax have lagged far behind what is known about $P$. falciparum (5). A milestone for the $P$. falciparum culture system was established by Trager and Jensen in 1976 (10), but was never established robustly for $P$. vivax. Although isolated reports documented long duration and continuous culture of $P$. vivax using stem cells or human reticulocytes, the resultant parasitemia was too low for reproducible drug inhibi-

Related Article: p. 2920

Conflict of interest: PA is a member of the board of directors of Aeromics. DJS has founding ownership equity interest in AliquantumRx, which seeks to develop cethromycin for malaria liver stages, and is a named inventor to Johns Hopkins-assigned patents for malaria diagnosis (USP 7,270,948; USP 9,568,471), angiogenesis inhibitors (USP $9,642,865)$, and provisional pending patents for cethromycin (US15/506,445 and 62/942,508) Copyright: (c) 2020, American Society for Clinical Investigation.

Reference information: / Clin Invest. 2020;130(6):2800-2802. https://doi.org/10.1172/JCl135794.

tion experiments, basic biochemistry, or ability to produce viable gametocytes (sexual stages) infectious for mosquitoes (1114). In contrast, the bench culture system for $P$. falciparum allows multiple mosquitoes to feed at once through a blood-filled membrane and achieves a nearly 100\% infection rate.

\section{Past and present controlled human malaria infections}

Blood-stage-induced human malaria infections have been brought back to life for research purposes after a long dormancy following World War II. Julius Wagner-Jauregg was awarded the 1927 Nobel Prize in Physiology or Medicine for discovering that high malarial fevers cured patients suffering from dementia-associated, end-stage syphilis. Malaria therapy was lethal in up to $15 \%$ of patients, especially when $P$. falciparum was used. $P$. vivax was preferred to greatly reduce risk of death. Malaria therapy spread throughout the world, requiring mosquitoes to propagate the parasite instead of direct blood transmission from patient to patient. In the 1930 s to 1960 s, dozens of isolates (strains) were used in the United States and Europe for malariotherapy. While the differing isolates induced variable fever cycles, relapse rates and duration, and rates of infection to mosquitoes $(15,16)$, archived studies from the United States show that the McCoy and St. Elizabeth $P$. vivax strains were most commonly used with success (16)

Mark Boyd looked at 46 patients who were infected with the $P$. vivax McCoy strain, and identified patients as poor or good infectors. Mosquito infection rates were $50 \%$ with detectable gametocytes by blood film (17). Boyd and Kitchen directly compared $P$. falciparum and $P$. vivax gametocytes, noting that individual $P$. vivax gametocytes transmit to Anopheles quadrimaculatus about ten times more effectively when compared with the $P$. falciparum gametocytes. Submicrosco- 
pic (blood film negative) $P$. vivax gametocytes infected $30 \%$ of mosquitoes, whereas submicroscopic $P$. falciparum gametocytes or even larger amounts (up to 25 gametocytes $/ \mu \mathrm{L}$ ) infected zero mosquitoes. In the range of 26 to 100 gametocytes/ $\mu \mathrm{L}$, $P$. vivax infection rates were $50 \%$, whereas that range in $P$. falciparum resulted in low mosquito infection rates (only 10\%) (18).

The expertise of malariotherapy was utilized for malaria drug and vaccine research during World War II when thousands of drugs, including chloroquine and primaquine, were screened in animals and humans. After WWII, human malaria studies ended, with research on malaria waning during the eradication efforts in the 1950s.

Modern day investigators first recreated human liver-stage infections by allowing $P$. falciparum-infected mosquitoes to bite subjects then testing the efficacy of liver-stage drugs and vaccines. As soon as blood stages appeared, the infections were cured, long before viable gametocytes achieved levels high enough to infect mosquitoes. A few studies used $P$. vivax liver-stage infection to look at relapse of dormant $P$. vivax.

Recently, a team of Australians led by James McCarthy repioneered infected human blood-stage $P$. falciparum infections based on careful screening for other human and animal pathogens and cryopreservation of large volumes of blood from an infected patient donor to look at drug and vaccine efficacy. Because of $P$. falciparum lethal outcome and the known delay of sexual-stage gametocyte appearance about a week after microscopic asexual stages, the ability to produce a $P$. falciparum model for human infection of mosquitoes is still a hurdle $(19,20)$.

The Australian investigators partially overcame the $P$. falciparum hurdle by using intermediate piperaquine doses to decrease asexual stages while preserving gametocytes. In the study, most subjects infected mosquitoes that had fed at any time over three weeks in $P$. falciparum blood-stage infection (21). In a follow-up study, combined drug regimens to cure asexual stages while preserving sexual stage gametocytes controlled malaria infection that was initiated by sporozoites; scarcely any mosquitoes became infected. In another study, researchers evaluated a malaria drug candidate (dihydroorotate dehydrogenase inhibitor) and noted that a dose of less than 1 gametocyte $/ \mu \mathrm{L}$ failed to infect the thousand mosquitoes tested (22). This $P$. falciparum human model is not robust!

In the first induced blood-stage infection reported with $P$. vivax acquired from the Solomon Islands in 2013, two subjects were followed without mosquito feeds. Both sustained gametocyte levels of over 1 gametocyte $/ \mu \mathrm{L}$ for two to three days before treatment on day 14 (23). In work published in 2016 for six volunteers, the initial $P$. vivax inoculum from the same Solomon Islands isolate was about six parasites per person. Later, the gametocyte level was less than 1 gametocyte $/ \mu \mathrm{L}$ at peak parasitemia, and infections to mosquitoes were scored by direct visualization of oocysts, which was subject to interpretation and gave mixed results.

In this issue of the JCI, Collins, McCarthy, and colleagues used a new $P$. vivax isolate from India (HMP013) that was inoculated at the higher density of 564 parasites per person, which is almost 100 times more than the six parasites per person in the previous 2016 cohort (24). The peak gametocyte density was 47 gametocytes $/ \mu \mathrm{L}$ in this study, shown by a revised PCR assay, yielding more than 300 times the gametocytes than with the Solomon Island isolate. Notably, $70 \%$ of the participants were susceptible to infected mosquitoes at peak gametocytemia. The researchers obtained pooled blood from participants and using a centrifugation gradient, enriched it ten-fold for gametocytes. The mosquito infection rate increased to $97 \%$, similar to that obtained for $P$. falciparum, when mosquitos were fed gametocyte-cultured blood through a membrane feeding assay. (24).

How do these infection rates compare with the rates of mosquitoes that fed on people with naturally acquired $P$. falciparum and $P$. vivax in recent years, as well as to those obtained from mosquito feeds on malariotherapy for syphilis studies or WWII research studies? Children with subclinical and clinical $P$. falciparum malaria present with fewer than 10 gametocytes/ $\mu \mathrm{L}$ of blood, which can infect $0.1 \%$ to $5 \%$ of mosquitoes. When there is a higher density of gametocytes, in the range of 100 to 1000 gametocytes $/ \mu \mathrm{L}$, the amount of mosquitoes that become infect- ed jumps to $10 \%$ to $20 \%$ (25). More than 1000 gametocytes/ $\mu \mathrm{L}$ is rare in human subjects. Notably, $P$. vivax mosquitoes fed on gametocytemic patients yielded an infection rate close to $5 \%$ (24).

\section{Conclusions}

For malaria therapy, doctors infecting mosquitoes was a means to continue propagation of the isolate for future syphilis patients. For modern day malariologists, natural infections are a means for testing the critical human-to-mosquito bottleneck with drugs or vaccines. The most recent study by Collins and colleagues from the McCarthy team marks an important advance in the quest to study and defeat the nefarious human parasite P. vivax (24).

\section{Acknowledgments}

PA and DJS are supported by Bloomberg Philanthropies. DJS is also supported by NIH R01 AI111962 and R21 TR001737.

Address correspondence to: David J. Sullivan, Johns Hopkins Bloomberg School of Public Health, 615 North Wolfe Street, Baltimore, Maryland 21205, USA. Phone: 410.502.2522; Email: dsulliv7@jhmi.edu.

1. WHO. World Malaria Report 2019. Geneva, Switzerland:WHO; 2019. http://www.who.int/ malaria/publications/world-malariareport-2019/en. Updated December 4, 2019. Accessed March 30, 2020.

2. Price RN, Tjitra E, Guerra CA, Yeung S, White NJ, Anstey NM. Vivax malaria: neglected and not benign. Am J Trop Med Hyg. 2007; 77(6 Suppl):79-87

3. Howes RE, et al. Global epidemiology of Plasmodium vivax. Am J Trop Med Hyg. 2016; 95(6 Suppl):15-34.

4. Battle KE, et al. Mapping the global endemicity and clinical burden of Plasmodium vivax, 200017: a spatial and temporal modelling study. Lancet. 2019;394(10195):332-343.

5. Baird JK. Evidence and implications of mortality associated with acute Plasmodium vivax malaria. Clin Microbiol Rev. 2013;26(1):36-57.

6. Price RN, Douglas NM, Anstey NM. New developments in Plasmodium vivax malaria: severe disease and the rise of chloroquine resistance. Curr Opin Infect Dis. 2009;22(5):430-435.

7. Neafsey DE, et al. The malaria parasite Plasmodium vivax exhibits greater genetic diversity than Plasmodium falciparum. Nat Genet. 2012;44(9):1046-1050.

8. Battle KE, et al. Geographical variation in Plasmodium vivax relapse. Malar J. 2014;13:144

9. Lover AA, Coker RJ. Quantifying effect of geographic location on epidemiology of Plasmodium vivax malaria. Emerging Infect Dis. 
2013;19(7):1058-1065.

10. Trager W, Jensen JB. Human malaria parasites in continuous culture. Science. 1976;193(4254):673-675.

11. Thomson-Luque R, Adams JH, Kocken CHM, Pasini EM. From marginal to essential: the golden thread between nutrient sensing, medium composition and Plasmodium vivax maturation in in vitro culture. Malar J. 2019;18(1):344.

12. Bermúdez M, Moreno-Pérez DA, ArévaloPinzón G, Curtidor H, Patarroyo MA. Plasmodium vivax in vitro continuous culture: the spoke in the wheel. Malar J. 2018;17(1):301.

13. Thomson-Luque R, Shaw Saliba K, Kocken CHM, Pasini EM. A continuous, long-term Plasmodium vivax in vitro blood-stage culture: what are we missing? Trends Parasitol. 2017;33(12):921-924.

14. Shaw-Saliba K, et al. Insights into an optimization of Plasmodium vivax Sal-1 in vitro culture: the aotus primate model. PLoS Negl Trop Dis. 2016;10(7):e0004870.
15. White NJ. Determinants of relapse periodicity in Plasmodium vivax malaria. Malar J. 2011;10:297.

16. Boyd M. On strains or races of the malaria parasites. Am J Trop Med Hyg. 1940;20(1):69-80.

17. Boyd M. On the varying infectiousness of different patients infected with vivax malaria. $A m J$ Trop Med Hyg. 1942;22(1):73-81.

18. Boyd M, Kitchen S. On the infectiousness of patients infected with Plasmodium vivax and Plasmodium falciparum. Am J Trop Med Hyg. 1937;17(2):253-262.

19. Farid R, Dixon MW, Tilley L, McCarthy JS. Initiation of gametocytogenesis at very low parasite density in Plasmodium falciparum infection. J Infect Dis. 2017;215(7):1167-1174

20. Pasay CJ, et al. Piperaquine monotherapy of drug-susceptible Plasmodium falciparum infection results in rapid clearance of parasitemia but is followed by the appearance of gametocytemia. J Infect Dis. 2016;214(1):105-113.

21. Collins KA, et al. A controlled human malaria infection model enabling evaluation of transmission-blocking interventions. JClin Invest . 2018;128(4):1551-1562.

22. Collins KA, et al. DSM265 at 400 milligrams clears asexual stage parasites but not mature gametocytes from the blood of healthy subjects experimentally infected with Plasmodium falciparum. Antimicrob Agents Chemother. 2019;63(4):e01837-18.

23. McCarthy JS, et al. Experimentally induced blood-stage Plasmodium vivax infection in healthy volunteers. J Infect Dis. 2013;208(10):1688-1694.

24. Collins K, et al. A Plasmodium vivax experimental human 1 infection model for evaluating efficacy of 2 interventions. JClin Invest. 2020;130(6):2920-2927.

25. Bousema T, Drakeley C. Epidemiology and infectivity of Plasmodium falciparum and Plasmodium vivax gametocytes in relation to malaria control and elimination. Clin Microbiol Rev. 2011;24(2):377-410 